\title{
Existence and Uniqueness of Solutions for Singular Higher Order Continuous and Discrete Boundary Value Problems
}

\author{
Chengjun Yuan, ${ }^{1,2}$ Daqing Jiang, ${ }^{1}$ and You Zhang ${ }^{1}$ \\ ${ }^{1}$ School of Mathematics and Statistics, Northeast Normal University, Changchun 130024, Jilin, China \\ ${ }^{2}$ School of Mathematics and Computer, Harbin University, Harbin 150086, Heilongiang, China \\ Correspondence should be addressed to Chengjun Yuan, ycj7102@163.com
}

Received 4 July 2007; Accepted 31 December 2007

Recommended by Raul Manasevich

By mixed monotone method, the existence and uniqueness are established for singular higher-order continuous and discrete boundary value problems. The theorems obtained are very general and complement previous known results.

Copyright $(0) 2008$ Chengjun Yuan et al. This is an open access article distributed under the Creative Commons Attribution License, which permits unrestricted use, distribution, and reproduction in any medium, provided the original work is properly cited.

\section{Introduction}

In recent years, the study of higher-order continuous and discrete boundary value problems has been studied extensively in the literature (see [1-17] and their references). Most of the results told us that the equations had at least single and multiple positive solutions.

Recently, some authors have dealt with the uniqueness of solutions for singular higherorder continuous boundary value problems by using mixed monotone method, for example, see $[6,14,15]$. However, there are few works on the uniqueness of solutions for singular discrete boundary value problems.

In this paper, we state a unique fixed point theorem for a class of mixed monotone operators, see $[6,14,18]$. In virtue of the theorem, we consider the existence and uniqueness of solutions for the following singular higher-order continuous and discrete boundary value problems (1.1) and (1.2) by using mixed monotone method. We first discuss the existence and uniqueness of solutions for the following singular higher-order continuous boundary value problem

$$
\begin{gathered}
y^{(n)}(t)+\lambda q(t)(g(y)+h(y))=0, \quad 0<t<1, \quad \lambda>0, \\
y^{(i)}(0)=y^{(n-2)}(1)=0, \quad 0 \leq i \leq n-2,
\end{gathered}
$$


where $n \geq 2, q(t) \in C((0,1),(0,+\infty)), g:[0,+\infty) \rightarrow[0,+\infty)$ is continuous and nondecreasing; $h:(0,+\infty) \rightarrow(0,+\infty)$ is continuous and nonincreasing, and $h$ may be singular at $y=0$.

Next, we consider the existence and uniqueness of solutions for the following singular higher-order discrete boundary value problem

$$
\begin{gathered}
\Delta^{n} y(i)+\lambda q(i+n-1)(g(y(i+n-1))+h(y(i+n-1)))=0, \quad i \in N=\{0,1,2, \ldots, T-1\}, \lambda>0, \\
\Delta^{k} y(0)=\Delta^{n-2} y(T+1)=0, \quad 0 \leq k \leq n-2,
\end{gathered}
$$

where $n \geq 2, N^{+}=\{0,1,2, \ldots, T+n\}, q(i) \in C\left(N^{+},(0,+\infty)\right), g:[0,+\infty) \rightarrow[0,+\infty)$ is continuous and nondecreasing; $h:(0,+\infty) \rightarrow(0,+\infty)$ is continuous and nonincreasing, and $h$ may be singular at $y=0$. Throughout this paper, the topology on $N^{+}$will be the discrete topology.

\section{Preliminaries}

Let $P$ be a normal cone of a Banach space $E$, and $e \in P$ with $\|e\| \leq 1, e \neq \theta$. Define

$$
Q_{e}=\{x \in P \mid x \neq \theta \text {, there exist constants } m, M>0 \text { such that } m e \leq x \leq M e\} .
$$

Now we give a definition (see [18]).

Definition 2.1 (see [18]). Assume $A: Q_{e} \times Q_{e} \rightarrow Q_{e}$. $A$ is said to be mixed monotone if $A(x, y)$ is nondecreasing in $x$ and nonincreasing in $y$, that is, if $x_{1} \leq x_{2}\left(x_{1}, x_{2} \in Q_{e}\right)$ implies $A\left(x_{1}, y\right) \leq$ $A\left(x_{2}, y\right)$ for any $y \in Q_{e}$, and $y_{1} \leq y_{2}\left(y_{1}, y_{2} \in Q_{e}\right)$ implies $A\left(x, y_{1}\right) \geq A\left(x, y_{2}\right)$ for any $x \in Q_{e}$. $x^{*} \in Q_{e}$ is said to be a fixed point of $A$ if $A\left(x^{*}, x^{*}\right)=x^{*}$.

Theorem 2.2 (see $[6,14]$ ). Suppose that $A: Q_{e} \times Q_{e} \rightarrow Q_{e}$ is a mixed monotone operator and $\exists$ a constant $\alpha, 0 \leq \alpha<1$, such that

$$
A\left(t x, \frac{1}{t} y\right) \geq t^{\alpha} A(x, y), \quad \text { for } x, y \in Q_{e}, 0<t<1 .
$$

Then $A$ has a unique fixed point $x^{*} \in Q_{e}$. Moreover, for any $\left(x_{0}, y_{0}\right) \in Q_{e} \times Q_{e}$,

$$
x_{n}=A\left(x_{n-1}, y_{n-1}\right), \quad y_{n}=A\left(y_{n-1}, x_{n-1}\right), \quad n=1,2, \ldots,
$$

satisfy

$$
x_{n} \longrightarrow x^{*}, \quad y_{n} \longrightarrow x^{*},
$$

where

$$
\left\|x_{n}-x^{*}\right\|=o\left(1-r^{\alpha^{n}}\right), \quad\left\|y_{n}-x^{*}\right\|=o\left(1-r^{\alpha^{n}}\right),
$$

$0<r<1, r$ is a constant from $\left(x_{0}, y_{0}\right)$.

Theorem 2.3 (see $[6,14,18]$ ). Suppose that $A: Q_{e} \times Q_{e} \rightarrow Q_{e}$ is a mixed monotone operator and $\exists a$ constant $\alpha \in(0,1)$ such that $(2.2)$ holds. If $x_{\lambda}^{*}$ is a unique solution of equation

$$
A(x, x)=\lambda x, \quad(\lambda>0)
$$

in $Q_{e}$, then $\left\|x_{\lambda}^{*}-x_{\lambda_{0}}^{*}\right\| \rightarrow 0, \lambda \rightarrow \lambda_{0}$. If $0<\alpha<1 / 2$, then $0<\lambda_{1}<\lambda_{2}$ implies $x_{\lambda_{1}}^{*} \geq x_{\lambda_{2}}^{*}, x_{\lambda_{1}}^{*} \neq x_{\lambda_{2}}^{*}$, and

$$
\lim _{\lambda \rightarrow+\infty}\left\|x_{\lambda}^{*}\right\|=0, \quad \lim _{\lambda \rightarrow 0^{+}}\left\|x_{\lambda}^{*}\right\|=+\infty .
$$




\section{Uniqueness positive solution of differential equations (1.1)}

This section discusses singular higher-order boundary value problem (1.1). Throughout this section, we let $G(t, s)$ be the Green's function to $-y^{\prime \prime}=0, y(0)=y(1)=0$, we note that

$$
G(t, s)= \begin{cases}t(1-s), & 0 \leq t \leq s \leq 1, \\ s(1-t), & 0 \leq s \leq t \leq 1,\end{cases}
$$

and one can show that

$$
G(t, t) G(s, s) \leq G(t, s) \leq G(t, t), \quad \text { for } G(t, s) \leq G(s, s),(t, s) \in[0,1] \times[0,1] .
$$

Suppose that $y$ is a positive solution of (1.1). Let

$$
x(t)=y^{(n-2)}(t)
$$

from $y^{(i)}(0)=y^{(n-2)}(1)=0,0 \leq i \leq n-2$, and Taylor Formula, we define operator $T: C^{(2)}[0,1] \rightarrow$ $C^{(n)}[0,1]$, by

$$
\begin{gathered}
y(t)=T x(t)=\int_{0}^{t} \frac{(t-s)^{n-3}}{(n-3) !} x(s) d s, \quad \text { for } 3 \leq n, \\
y(t)=T x(t)=x(t), \quad \text { for } n=2 .
\end{gathered}
$$

Then we have

$$
\begin{gathered}
x^{(2)}(t)+\lambda f(t, T x(t))=0, \quad 0<t<1, \lambda>0, \\
x(0)=x(1)=0 .
\end{gathered}
$$

Then from (3.4), we have the next lemma.

Lemma 3.1. If $x(t)$ is a solution of (3.5), then $y(t)$ is a solution of (1.1).

Further, if $y(t)$ is a solution of (1.1), imply that $x(t)$ is a solution of (3.5).

Let $P=\{x \in C[0,1] \mid x(t) \geq 0$, for all $t \in[0,1]\}$. Obviously, $P$ is a normal cone of Banach space $C[0,1]$.

Theorem 3.2. Suppose that there exists $\alpha \in(0,1)$ such that

$$
\begin{gathered}
g(t x) \geq t^{\alpha} g(x), \\
h\left(t^{-1} x\right) \geq t^{\alpha} h(x),
\end{gathered}
$$

for any $t \in(0,1)$ and $x>0$, and $q \in C((0,1),(0, \infty))$ satisfies

$$
\int_{0}^{1}\left[s^{n-1}(n-2 s)\right]^{-\alpha} q(s) d s<+\infty .
$$

Then (1.1) has a unique positive solution $y_{\lambda}^{*}(t)$. And moreover, $0<\lambda_{1}<\lambda_{2}$ implies $y_{\lambda_{1}}^{*} \leq y_{\lambda_{2}}^{*}, y_{\lambda_{1}}^{*} \neq y_{\lambda_{2}}^{*}$. If $\alpha \in(0,1 / 2)$, then

$$
\lim _{\lambda \rightarrow 0^{+}}\left\|y_{\lambda}^{*}\right\|=0, \quad \lim _{\lambda \rightarrow+\infty}\left\|y_{\lambda}^{*}\right\|=+\infty
$$


Proof. Since (3.7) holds, let $t^{-1} x=y$, one has

$$
h(y) \geq t^{\alpha} h(t y)
$$

Then

$$
h(t y) \leq \frac{1}{t^{\alpha}} h(y), \quad \text { for } t \in(0,1), y>0 .
$$

Let $y=1$. The above inequality is

$$
h(t) \leq \frac{1}{t^{\alpha}} h(1), \quad \text { for } t \in(0,1) .
$$

From (3.7), (3.11), and (3.12), one has

$$
h\left(t^{-1} x\right) \geq t^{\alpha} h(x), \quad h\left(\frac{1}{t}\right) \geq t^{\alpha} h(1), \quad h(t x) \leq \frac{1}{t^{\alpha}} h(x), \quad h(t) \leq \frac{1}{t^{\alpha}} h(1), \text { for } t \in(0,1), x>0 .
$$

Similarly, from (3.6), one has

$$
g(t x) \geq t^{\alpha} g(x), \quad g(t) \geq t^{\alpha} g(1), \quad \text { for } t \in(0,1), x>0 .
$$

Let $t=1 / x, x>1$, one has

$$
g(x) \leq x^{\alpha} g(1), \quad \text { for } x \geq 1
$$

Let $e(t)=G(t, t)=t(1-t)$, and we define

$$
Q_{e}=\left\{x \in C[0,1] \mid \frac{1}{M} G(t, t) \leq x(t) \leq M G(t, t), t \in[0,1]\right\},
$$

where $M>1$ is chosen such that

$$
\begin{aligned}
M>\max \{ & {\left.\left[\lambda g(1) \int_{0}^{1} q(s) d s+\lambda h(1) \int_{0}^{1}\left(\frac{s^{n-1}(n-2 s)}{n !}\right)\right)^{-\alpha} q(s) d s\right]^{1 /(1-\alpha)}, } \\
& {\left.\left[\lambda g(1) \int_{0}^{1} G(s, s)\left(\frac{s^{n-1}(n-2 s)}{n !}\right)^{\alpha} q(s) d s+\lambda h(1) \int_{0}^{1} G(s, s) q(s) d s\right]^{-1 /(1-\alpha)}\right\} . }
\end{aligned}
$$

First, from (3.4) and (3.16), for any $x \in Q_{e}$, we have the following.

When $3 \leq n$,

$$
\begin{aligned}
\frac{1}{M} \frac{t^{n-1}(n-2 t)}{n !} & \leq \int_{0}^{t} \frac{1}{M} G(s, s) \frac{(t-s)^{n-3}}{(n-3) !} d s \leq T x(t) \\
& \leq \int_{0}^{t} M G(s, s) \frac{(t-s)^{n-3}}{(n-3) !} d s \leq M \frac{t^{n-1}(n-2 t)}{n !} \leq M, \quad \text { for } t \in[0,1]
\end{aligned}
$$


when $n=2$,

$$
\frac{1}{M} \frac{t^{n-1}(n-2 t)}{n !} \leq T x(t)=x(t) \leq M \frac{t(n-2 t)}{n !} \leq M, \quad \text { for } t \in[0,1]
$$

then

$$
\frac{1}{M} \frac{t^{n-1}(n-2 t)}{n !} \leq T x(t) \leq M \frac{t^{n-1}(n-2 t)}{n !} \leq M, \quad \text { for } t \in[0,1]
$$

For any $x, y \in Q_{e}$, we define

$$
A_{\lambda}(x, y)(t)=\lambda \int_{0}^{1} G(t, s) q(s)[g(T x(s))+h(T y(s))] d s, \quad \text { for } t \in[0,1]
$$

First, we show that $A_{\curlywedge}: Q_{e} \times Q_{e} \rightarrow Q_{e}$.

Let $x, y \in Q_{e}$, from (3.14), (3.15), and (3.20), we have

$$
g(T x(t)) \leq g(M) \leq M^{\alpha} g(1), \quad \text { for } t \in(0,1),
$$

and from (3.13), we have

$$
\begin{aligned}
h(T y(t)) & \leq h\left(\frac{1}{M} \frac{t^{n-1}(n-2 t)}{n !}\right) \leq\left[\frac{t^{n-1}(n-2 t)}{n !}\right]^{-\alpha} h\left(\frac{1}{M}\right) \\
& \leq M^{\alpha}\left[\frac{t^{n-1}(n-2 t)}{n !}\right]^{-\alpha} h(1), \quad \text { for } t \in(0,1) .
\end{aligned}
$$

Then, from (3.2), (3.21), (3.22) and (3.23), we have

On the other hand, for any $x, y \in Q_{e}$, from (3.13) and (3.14), we have

$$
\begin{gathered}
g(T x(t)) \geq g\left(\frac{1}{M} \frac{t^{n-1}(n-2 t)}{n !}\right) \geq\left(\frac{t^{n-1}(n-2 t)}{n !}\right)^{\alpha} g\left(\frac{1}{M}\right) \geq\left(\frac{t^{n-1}(n-2 t)}{n !}\right)^{\alpha} \frac{1}{M^{\alpha}} g(1) \\
h(T y(t)) \geq h(M)=h\left(\frac{1}{1 / M}\right) \geq \frac{1}{M^{\alpha}} h(1), \quad \text { for } t \in(0,1) .
\end{gathered}
$$

Thus, from (3.2), (3.21) and (3.24), we have

$$
\begin{aligned}
& A_{\lambda}(x, y)(t) \\
& \quad \geq \lambda G(t, t)\left\{\int_{0}^{1} G(s, s) q(s) M^{-\alpha}\left[\frac{s^{n-1}(n-2 s)}{n !}\right]^{\alpha} g(1) d s+\int_{0}^{1} G(s, s) q(s) M^{-\alpha} h(1) d s\right. \\
& \quad \geq \frac{1}{M} G(t, t), \quad \text { for } t \in[0,1] .
\end{aligned}
$$

So, $A_{\curlywedge}$ is well defined and $A_{\curlywedge}\left(Q_{e} \times Q_{e}\right) \subset Q_{e}$. 
Next, for any $l \in(0,1)$, one has

$$
\begin{aligned}
A_{\curlywedge}\left(l x, l^{-1} y\right)(t) & =\lambda \int_{0}^{1} G(t, s) q(s)\left[g(l T x(s))+h\left(l^{-1} T y(s)\right)\right] d s \\
& \geq \lambda \int_{0}^{1} G(t, s) q(s)\left[l^{\alpha} g(T x(s))+l^{\alpha} h(T y(s))\right] d s \\
& =l^{\alpha} A_{\lambda}(x, y)(t), \quad \text { for } t \in[0,1] .
\end{aligned}
$$

So the conditions of Theorems 2.2 and 2.3 hold. Therefore, there exists a unique $x_{\lambda}^{*} \in Q_{e}$ such that $A_{\lambda}\left(x^{*}, x^{*}\right)=x_{\lambda}^{*}$. It is easy to check that $x_{\lambda}^{*}$ is a unique positive solution of (3.5) for given $\lambda>0$. Moreover, Theorem 2.3 means that if $0<\lambda_{1}<\lambda_{2}$, then $x_{\lambda_{1}}^{*}(t) \leq x_{\lambda_{2}}^{*}(t), x_{\lambda_{1}}^{*}(t) \neq x_{\lambda_{2}}^{*}(t)$ and if $\alpha \in(0,1 / 2)$, then

$$
\lim _{\lambda \rightarrow 0^{+}}\left\|x_{\lambda}^{*}\right\|=0, \quad \lim _{\lambda \rightarrow+\infty}\left\|x_{\lambda}^{*}\right\|=+\infty
$$

Next, from Lemma 3.1 and (3.4), we get that $y_{\lambda}^{*}=T x_{\lambda}^{*}$ is a unique positive solution of (1.1) for given $\lambda>0$. Moreover, if $0<\lambda_{1}<\lambda_{2}$, then $y_{\lambda_{1}}^{*}(t) \leq y_{\lambda_{2}}^{*}(t), y_{\lambda_{1}}^{*}(t) \neq y_{\lambda_{2}}^{*}(t)$ and if $\alpha \in(0,1 / 2)$, then

$$
\lim _{\lambda \rightarrow 0^{+}}\left\|y_{\lambda}^{*}\right\|=0, \quad \lim _{\lambda \rightarrow+\infty}\left\|y_{\lambda}^{*}\right\|=+\infty
$$

This completes the proof.

Example 3.3. Consider the following singular boundary value problem:

$$
\begin{gathered}
y^{(n)}(t)+\lambda\left(\mu y^{a}(t)+y^{-b}(t)\right)=0, \quad t \in[0,1], \\
y^{(i)}(0)=y^{(n-2)}(1)=0, \quad 0 \leq i \leq n-2,
\end{gathered}
$$

where $\lambda, a, b>0, \mu \geq 0, \max \{a, b\}<1 /(n-1)$.

Applying Theorem 3.2, let $\alpha=\max \{a, b\}<1 /(n-1), q(t)=1, g(y)=\mu y^{a}, h(y)=y^{-b}$, then

$$
\begin{gathered}
g(t y) \geq t^{\alpha} g(y), \quad h\left(t^{-1}\right) \geq t^{\alpha} h(y), \\
\int_{0}^{1}\left[s^{n-1}(n-2 s)\right]^{-\alpha} d s<+\infty
\end{gathered}
$$

Thus all conditions in Theorem 3.2 are satisfied. We can find (3.29) has a unique positive solution $y_{\lambda}^{*}(t)$. In addition, $0<\lambda_{1}<\lambda_{2}$ implies $y_{\lambda_{1}}^{*} \leq y_{\lambda_{2}}^{*}, y_{\lambda_{1}}^{*} \neq y_{\lambda_{2}}^{*}$. If $\alpha=\max \{a, b\} \in(0,1 / 2)$, then

$$
\lim _{\lambda \rightarrow 0^{+}}\left\|y_{\lambda}^{*}\right\|=0, \quad \lim _{\lambda \rightarrow+\infty}\left\|y_{\lambda}^{*}\right\|=+\infty
$$




\section{Uniqueness positive solution of difference equations (1.2)}

This section discusses singular higher-order boundary value problem (1.2). Throughout this section, we let $K(i, j)$ be Green's function to $-\Delta^{2} y(i)+u(i+1)=0, i \in N, y(0)=y(T+1)=0$, we note that

$$
K(i, j)= \begin{cases}\frac{j(T+1-i)}{T+1}, & 0 \leq j \leq i-1, \\ \frac{i(T+1-j)}{T+1}, & i \leq j \leq T+1,\end{cases}
$$

and one can show that

$$
K(i, i) \geq K(i, j), \quad K(j, j) \geq K(i, j), \quad K(i, j) \geq \frac{K(i, i)}{T+1}, \quad \text { for } 0 \leq i \leq T+1,1 \leq j \leq T .
$$

Suppose that $y$ is a positive solution of (1.2). Let

$$
x(i)=\Delta^{n-2} y(i), \quad \text { for } 0 \leq i \leq T+1 .
$$

From $\Delta^{i} y(0)=\Delta^{n-2} y(T+1)=0,0 \leq i \leq n-2$, and $\Delta^{m} y(i-1)=\Delta^{m-1} y(i)-\Delta^{m-1} y(i-1)$, so we define operator $T$, by

$$
T x(i)=y(i+n-1)=\sum_{l=1}^{i+1} C_{i-l+n-1}^{n-2} x(l), \quad \text { for } 0 \leq i \leq T .
$$

Then

$$
\begin{gathered}
\Delta^{2} x(i)+\lambda F(i+n-1, T x(i))=0, \quad 0 \leq i \leq T-1, \lambda>0, \\
x(0)=x(T+1)=0 .
\end{gathered}
$$

Lemma 4.1. If $x(i)$ is a solution of (4.5), then $y(i)$ is a solutionn of (1.2).

Proof. Since we remark that $x(i)$ is a solution of (4.5), if and only if

$$
x(i)=\sum_{j=1}^{T} K(i, j) \lambda F(j+n-1, T x(j)), \quad \text { for } 0 \leq i \leq T+1 .
$$

Let

$$
T x(i)=y(i+n-1), \quad \text { for } 0 \leq i \leq T .
$$

From (4.4) we find $\Delta^{i} y(0)=\Delta^{n-2} y(T+1)=0,0 \leq i \leq n-2$, and $x(i)=\Delta^{n-2} y(i)$, so that $y(i)$ is a solution of (1.2).

Further, if $y(i)$ is a solution of (1.2), imply that $x(i)$ is a solution of (4.5).

Let $P=\left\{x \in C\left(N^{+},[0,+\infty)\right) \mid x(i) \geq 0\right.$, for all $\left.i \in N^{+}\right\}$. Obviously, $P$ is a normal cone of Banach space $C\left(N^{+},[0,+\infty)\right)$. 
Theorem 4.2. Suppose that there exists $\alpha \in(0,1)$ such that

$$
\begin{gathered}
g(t x) \geq t^{\alpha} g(x), \\
h\left(t^{-1} x\right) \geq t^{\alpha} g(x),
\end{gathered}
$$

for any $t \in(0,1)$ and $x>0$, and $q \in C\left(N^{+},(0, \infty)\right)$.

Then (1.2) has a unique positive solution $y_{\lambda}^{*}(i)$. And moreover, $0<\lambda_{1}<\lambda_{2}$ implies $y_{\lambda_{1}}^{*} \leq y_{\lambda_{2}}^{*}$ $y_{\lambda_{1}}^{*} \neq y_{\lambda_{2}}^{*}$. If $\alpha \in(0,1 / 2)$, then

$$
\lim _{\lambda \rightarrow 0^{+}}\left\|y_{\lambda}^{*}\right\|=0, \quad \lim _{\lambda \rightarrow+\infty}\left\|y_{\lambda}^{*}\right\|=+\infty
$$

Proof. The proof is the same as that of Theorem 3.2, from (4.12) and (4.13), one has

$$
\begin{aligned}
h\left(t^{-1} x\right) \geq t^{\alpha} h(x), \quad & h\left(\frac{1}{t}\right) \geq t^{\alpha} h(1), \quad h(t x) \leq \frac{1}{t^{\alpha}} h(x), \quad h(t) \leq \frac{1}{t^{\alpha}} h(1), \quad \text { for } t \in(0,1), x>0 ; \\
& g(t x) \geq t^{\alpha} g(x), g(t) \geq t^{\alpha} g(1), \quad \text { for } t \in(0,1), x>0 . \\
& g(t x) \geq t^{\alpha} g(x), g(t) \geq t^{\alpha} g(1), \quad \text { for } t \in(0,1), x>0 .
\end{aligned}
$$

Let $t=1 / x, x>1$, one has

$$
g(x) \leq x^{\alpha} g(1), \quad \text { for } x \geq 1
$$

Let $e(i)=K(i, i) /(T+1)$, and we define

$$
Q_{e}=\left\{x \in P \mid \frac{1}{M} e(i) \leq x(i) \leq M e(i), \text { for } 0 \leq i \leq T+1\right\},
$$

where $M>1$ is chosen such that

$$
\begin{aligned}
M>\max \{ & {\left[\lambda(T+1) g(1) \sum_{j=1}^{T} q(j+n-1)\left(\sum_{l=1}^{j+1} C_{j-l+n-1}^{n-2}\right)^{\alpha}\right.} \\
& \left.+\lambda(T+1)^{1+\alpha} h(1) \sum_{j=1}^{T} K^{-\alpha}(j, j) q(j+n-1)\right]^{1 /(1-\alpha)} ; \\
& {\left.\left[\lambda g(1) \sum_{j=1}^{T} q(j+n-1)\left(\frac{K(j, j)}{T+1}\right)^{\alpha}+\lambda h(1) \sum_{j=1}^{T} q(j+n-1)\left(\sum_{l=1}^{j+1} C_{j-l+n-1}^{n-2}\right)^{-\alpha}\right]^{-1 /(1-\alpha)}\right\} . }
\end{aligned}
$$

From (4.4) and (4.13), for any $x \in Q_{e}$, we have

$$
\frac{1}{M} e(j) \leq T x(j)=\sum_{l=1}^{j+1} C_{j-l+n-1}^{n-2} x(l) \leq M e(j) \sum_{l=1}^{j+1} C_{j-l+n-1}^{n-2}, \quad \text { for } 0 \leq j \leq T .
$$


For any $x, y \in Q_{e}$, we define

$$
A_{\lambda}(x, y)(i)=\lambda \sum_{j=1}^{T} K(i, j) q(j+n-1)[g(T x(j))+h(T y(j))], \quad \text { for } 0 \leq i \leq T+1 .
$$

First we show that $A_{\curlywedge}: Q_{e} \times Q_{e} \rightarrow Q_{e}$.

Let $x, y \in Q_{e}$, from (4.11) and (4.12), we have

$$
g(T x(j)) \leq g\left(M e(j) \sum_{l=1}^{j+1} C_{j-l+n-1}^{n-2}\right) \leq g\left(M \sum_{l=1}^{j+1} C_{j-l+n-1}^{n-2}\right) \leq M^{\alpha}\left(\sum_{l=1}^{j+1} C_{j-l+n-1}^{n-2}\right)^{\alpha} g(1), \quad \text { for } 1 \leq j \leq T,
$$

and from (4.10), we have

$$
h(T y(j)) \leq h\left(\frac{1}{M} e(j)\right) \leq e^{-\alpha}(j) h\left(\frac{1}{M}\right) \leq M^{\alpha} e^{-\alpha}(j) h(1), \quad \text { for } 1 \leq j \leq T .
$$

Then, from (4.2) and the above, we have

$$
\begin{aligned}
A_{\lambda}(x, y)(i) \leq \lambda K(i, i) \sum_{j=1}^{T} q(j+n-1)[g(T x(j))+(T+1) h(T y(j))] \\
\leq e(i) M^{\alpha} \lambda(T+1)\left[g(1) \sum_{j=1}^{T} q(j+n-1)\left(\sum_{l=1}^{j+1} C_{j-l+n-1}^{n-2}\right)^{\alpha}\right. \\
\left.+h(1) \sum_{j=1}^{T} e^{-\alpha}(j) q(j+n-1)\right] \\
\leq e(i) M^{\alpha} \lambda(T+1)\left[g(1) \sum_{j=1}^{T} q(j+n-1)\left(\sum_{l=1}^{j+1} C_{j-l+n-1}^{n-2}\right)^{\alpha}\right. \\
\left.\quad+h(1) \sum_{j=1}^{T}\left(\frac{K(j, j)}{T+1}\right)^{-\alpha} q(j+n-1)\right] \\
\leq M e(i), \quad \text { for } 0 \leq i \leq T+1 .
\end{aligned}
$$

On the other hand, for any $x, y \in Q_{e}$, from (4.10) and (4.12), we have

$$
\begin{gathered}
g(x(j)) \geq g\left(\frac{1}{M} e(j)\right) \geq e^{\alpha}(j) \frac{1}{M^{\alpha}} g(1), \quad \text { for } 1 \leq j \leq T, \\
h(y(j)) \geq h\left(M e(j) \sum_{l=1}^{j+1} C_{j-l+n-1}^{n-2}\right) \geq M^{-\alpha}\left(\sum_{l=1}^{j+1} C_{j-l+n-1}^{n-2}\right)^{-\alpha} h(1), \quad \text { for } 1 \leq j \leq T .
\end{gathered}
$$

Thus, from (4.2) and (4.16), we have

$$
\begin{aligned}
A_{\lambda}(x, y)(i) & \geq \operatorname{le}(i)\left[\sum_{j=0}^{T} q(j+n-1) g(T x(j))+\sum_{j=0}^{T} q(j+n-1) h(T y(j))\right] \\
& \geq \operatorname{le}(i) M^{-\alpha}\left[g(1) \sum_{j=0}^{T} q(j)\left(\frac{K(i, i)}{T+1}\right)^{\alpha}+h(1) \sum_{j=0}^{T} q(j+n-1)\left(\sum_{l=1}^{j+1} C_{j-l+n-1}^{n-2}\right)^{-\alpha}\right] \\
& \geq \frac{1}{M} e(i), \quad \text { for } 0 \leq i \leq T+1 .
\end{aligned}
$$


So, $A_{\curlywedge}$ is well defined and $A_{\curlywedge}\left(Q_{e} \times Q_{e}\right) \subset Q_{e}$.

Next, for any $l \in(0,1)$, one has

$$
\begin{aligned}
A_{\curlywedge}\left(l x, l^{-1} y\right)(i) & =\lambda \sum_{j=1}^{T} K(i, j) q(j+n-1)\left[g(T(l x)(j))+h\left(T\left(l^{-1}\right) y(j)\right)\right] \\
& =\lambda \sum_{j=1}^{T} K(i, j) q(j+n-1)\left[g(l T x(j))+h\left(l^{-1} T y(j)\right)\right] \\
& \geq \lambda \sum_{j=1}^{T} K(i, j) q(j+n-1)\left[l^{\alpha} g(T x(j))+l^{\alpha} h(T y(j))\right] d s \\
& =l^{\alpha} A_{\curlywedge}(x, y)(i), \quad \text { for } 0 \leq i \leq T+1 .
\end{aligned}
$$

So the conditions of Theorems 2.2 and 2.3 hold. Therefore, there exists a unique $x_{\lambda}^{*} \in Q_{e}$ such that $A_{\lambda}\left(x^{*}, x^{*}\right)=x_{\lambda}^{*}$. It is easy to check that $x_{\lambda}^{*}$ is a unique positive solution of (4.5) for given $\lambda>0$. Moreover, Theorem 2.3 means that if $0<\lambda_{1}<\lambda_{2}$, then $x_{\lambda_{1}}^{*}(t) \leq x_{\lambda_{2}}^{*}(t), x_{\lambda_{1}}^{*}(t) \neq x_{\lambda_{2}}^{*}(t)$ and if $\alpha \in(0,1 / 2)$, then

$$
\lim _{\lambda \rightarrow 0^{+}}\left\|x_{\lambda}^{*}\right\|=0, \quad \lim _{\lambda \rightarrow+\infty}\left\|x_{\lambda}^{*}\right\|=+\infty .
$$

Next, on using Lemma 3.1, from (4.5), we get that $y_{\lambda}^{*}=T x_{\lambda}^{*}$ is a unique positive solution of (1.2) for given $\lambda>0$. Moreover, if $0<\lambda_{1}<\lambda_{2}$, then $y_{\lambda_{1}}^{*}(t) \leq y_{\lambda_{2}}^{*}(t), y_{\lambda_{1}}^{*}(t) \neq y_{\lambda_{2}}^{*}(t)$ and if $\alpha \in(0,1 / 2)$, then

$$
\lim _{l \rightarrow 0^{+}}\left\|y_{\lambda}^{*}\right\|=0, \quad \lim _{l \rightarrow+\infty}\left\|y_{\lambda}^{*}\right\|=+\infty
$$

This completes the proof.

Example 4.3. Consider the following singular boundary value problem:

$$
\begin{gathered}
\Delta^{n} y(i-1)+\lambda\left(\mu y^{a}(i)+y^{-b}(i)\right)=0, \quad i \in N, \\
\Delta^{i} y(0)=\Delta^{n-2} y(1)=0, \quad 0 \leq i \leq n-2,
\end{gathered}
$$

where $\lambda, a, b>0, \mu \geq 0, \max \{a, b\}<1$.

Let $q(i)=1, g(y)=\mu y^{a}, h(y)=y^{-b}, \alpha=\max \{a, b\}<1$, then

$$
g(t y) \geq t^{\alpha} g(y), \quad h\left(t^{-1} y\right) \geq t^{\alpha} h(y),
$$

thus all conditions in Theorem 4.2 are satisfied. We can find (4.26) has a unique positive solution $y_{\lambda}^{*}(t)$. In addition, $0<\lambda_{1}<\lambda_{2}$ implies $y_{\lambda_{1}}^{*} \leq y_{\lambda_{2}}^{*}, y_{\lambda_{1}}^{*} \neq y_{\lambda_{2}}^{*}$. If $\alpha=\max \{a, b\} \in(0,1 / 2)$, then

$$
\lim _{\lambda \rightarrow 0^{+}}\left\|y_{\lambda}^{*}\right\|=0, \quad \lim _{\lambda \rightarrow+\infty}\left\|y_{\lambda}^{*}\right\|=+\infty
$$




\section{Acknowledgments}

The work was supported by the National Natural Science Foundation of China (Grants no. 10571021 and 10701020). The work was supported by Subject Foundation of Harbin University (Grant no. HXK200714).

\section{References}

[1] Y. Guo and J. Tian, "Positive solutions of m-point boundary value problems for higher order ordinary differential equations," Nonlinear Analysis. Theory, Methods \& Applications, vol. 66, no. 7, pp. 1573-1586, 2007.

[2] D. Jiang, "Multiple positive solutions to singular boundary value problems for superlinear higherorder ODEs," Computers and Mathematics with Applications, vol. 40, no. 2-3, pp. 249-259, 2000.

[3] P. W. Eloe and J. Henderson, "Singular nonlinear boundary value problems for higher order ordinary differential equations," Nonlinear Analysis. Theory, Methods $\mathcal{E}$ Applications, vol. 17, no. 1, pp. 1-10, 1991.

[4] P. W. Eloe and J. Henderson, "Postive solutions for higher order differential equations," Journal of Differential Equations, vol. 3, pp. 1-8, 1995.

[5] C. J. Chyan and J. Henderson, "Positive solutions for singular higher order nonlinear equations," Differential Equations and Dynamical Systems, vol. 2, no. 2, pp. 153-160, 1994.

[6] X. Lin, D. Jiang, and X. Li, "Existence and uniqueness of solutions for singular $(k, n-k)$ conjugate boundary value problems," Computers \& Mathematics with Applications, vol. 52, no. 3-4, pp. 375-382, 2006.

[7] R. P. Agarwal and D. O'Regan, "Existence theory for single and multiple solutions to singular positone boundary value problems," Journal of Differential Equations, vol. 175, no. 2, pp. 393-414, 2001.

[8] P. R. Agarwal and D. O'Regan, "Singular discrete boundary value problems," Applied Mathematics Letters, vol. 12, no. 4, pp. 127-131, 1999.

[9] R. P. Agarwal and F.-H. Wong, "Existence of positive solutions for higher order difference equations," Applied Mathematics Letters, vol. 10, no. 5, pp. 67-74, 1997.

[10] Z. Du, C. Xue, and W. Ge, "Triple solutions for a higher-order difference equation," Journal of Inequalities in Pure and Applied Mathematics, vol. 6, no. 1, Article 10, pp. 1-11, 2005.

[11] P. J. Y. Wong and R. P. Agarwal, "On the existence of solutions of singular boundary value problems for higher order difference equations," Nonlinear Analysis. Theory, Methods \& Applications, vol. 28, no. 2, pp. 277-287, 1997.

[12] R. P. Agarwal and P. J. Y. Wong, "Existence of solutions for singular boundary problems for higher order differential equations," Rendiconti del Seminario Matemàtico e Fisico di Milano, vol. 65, pp. 249264, 1995.

[13] R. P. Agarwal and F.-H. Wong, "Existence of positive solutions for higher order difference equations," Applied Mathematics Letters, vol. 10, no. 5, pp. 67-74, 1997.

[14] X. Lin, D. Jiang, and X. Li, "Existence and uniqueness of solutions for singular fourth-order boundary value problems," Journal of Computational and Applied Mathematics, vol. 196, no. 1, pp. 155-161, 2006.

[15] Z. Zengqin, "Uniqueness of positive solutions for singular nonlinear second-order boundary-value problems," Nonlinear Analysis. Theory, Methods \& Applications, vol. 23, no. 6, pp. 755-765, 1994.

[16] R. P. Agarwal and I. Kiguradze, "Two-point boundary value problems for higher-order linear differential equations with strong singularities," Boundary Value Problems, vol. 2006, Article ID 83910, 32 pages, 2006.

[17] X. Hao, L. Liu, and Y. Wu, "Positive solutions for nonlinear $n$ th-order singular nonlocal boundary value problems," Boundary Value Problems, vol. 2007, Article ID 74517, 10 pages, 2007.

[18] D. Guo, The Order Methods in Nonlinear Analysis, Shandong Technical and Science Press, Jinan, China, 2000. 